\title{
Properties of Rough Interfaces in Superconductors with d-Wave Pairing
}

\author{
Alexander A. Golubov \\ Low Temperature Division, Department of Applied Physics, University of Twente, P.O. box 217, 7500 AE Enschede, The Netherlands \\ Mikhail Yu. Kupriyanov \\ Nuclear Physics Institute, Moscow State University, 119899 GSP Moscow, Russia
}

\begin{abstract}
Theoretical model of a rough interface in a superconductor with d-wave symmetry of the order parameter is proposed. The surface roughness is introduced by means of a surface layer with small electronic mean free path. The proximity effect between such a layer and a bulk $d$-wave superconductor is studied theoretically in the framework of the quasiclassical Eilenberger theory. It is shown that as a result of strong scattering in the interlayer the d-wave component of the order parameter near the interface is reduced while the s-wave component localized near the interface is generated. Angular and spatial structure of the pair potential and the electronic density of states near the interface is calculated. The interplay of the zeroenergy (midgap) and finite-energy bound states leads to peculiarities in the energy dependence of the angle-averaged density of states. We argue that the model is relevant for the description of rough interfaces in high $T_{c}$ superconductors. In the framework of the present approach we calculate the Josephson critical current for several types of junctions with rough interfaces.
\end{abstract}

\section{INTRODUCTION}

There is accumulating experimental evidence that the behavior of high temperature superconductors (HTS) can be understood in terms of the $d$-wave pairing scenario rather than in the conventional s-wave picture. On the other hand it is well known that the d-wave order parameter is strongly reduced by electron scattering at impurities and therefore can be formed only in clean materials. However, the condition of clean limit is not fulfilled in the vicinity of the grain boundaries or other HTS interfaces even if these materials are clean in the bulk. There are at least two reasons for that. The first is that quasiparticle reflection from realistic interfaces is diffusive rather than specular, thus providing isotropization in momentum space and suppression of the d-wave component of the order parameter. The second reason is the contamination of the material near interfaces as a result of fabrication process or electromigration in large scale application devices. Therefore the formation of a thin disordered layer near a HTS surfaces and interfaces is highly probable. An important question is whether or not superconducting correlations vanish in such a layer in the limit of small mean free path and what is the orbital structure of the superconducting state in the interface region.

Manuscript received September 14, 1998.

This work supported in part by INTAS Grant $93-790$ ext and by the Program for Russian-Dutch Research Cooperation (NWO).
Surface peculiarities in d-wave superconductors were extensively discussed in the framework of the theoretical models based on specular quasiparticle reflection from clean interfaces [1]-[4]. Zero- and finite-bias anomalies predicted in these papers were recently observed experimentally in [5], [6]. In this paper we focus on the problem of the anomalous proximity effect between a d-wave superconductor and a thin disordered layer in the limit of strong disorder. It is shown that an isotropic order parameter is nucleated in such a layer even in the absence of the subdominant pairing interaction in the s-wave channel. The spatially-resolved quasiparticle density of states is calculated. It is shown that zero- and finiteenergy peaks are present in the surface density of states in the d-wave region. Zero-energy peaks are fully smeared out in the disordered layer, which is in a peculiar gapless superconducting state.

\section{THE MODEL}

Two approaches to the study of surface roughness effects in unconventional superconductors were used previously. In the first one it is assumed that the interface consists of facets with random orientations compared to the crystallographic axes of the material [4]. According to the second approach, both sides of an ideal interface are coated by a so-called Ovchinnikov's thin disordered layer [3], [7, [8]. In the latter case the degree of disorder (or interface roughness) is measured by the ratio of the layer thickness $d$ to the quasiparticle mean free path in the layer $l$. Up to now both approaches were used to study the smearing of Andreev surface bound states by weak disorder. Here we will concentrate on the regime of strong disorder. We consider the surface or a weakly transparent barrier in a d-wave superconductor oriented normal to the crystallographic $a b$ plane. We assume that the crossover from the clean to the dirty limit takes place in a thin layer near the surface with mean free path $l$ and thickness $d<\sqrt{\xi_{0} l}$, where $\xi_{0}$ is the coherence length of the bulk material.

To study the proximity effect at the interface we use the quasiclassical Eilenberger equations [9] with impurity scattering taken in the Born limit. As is shown in [10], the problem is reduced to the solution of the Eilenberger equations within the clean $d$-wave superconductor $(x>0)$

$$
\begin{aligned}
& k^{2} \Phi_{+}^{\prime \prime}-\Phi_{+}=-\Delta g / \omega, \quad k=v|\cos \theta| / 2 \omega \\
& g^{\prime}=-\Delta \Phi_{+}^{\prime} / \omega
\end{aligned}
$$


with the condition in the bulk of a superconductor

$$
\begin{aligned}
& \Phi_{+}(x=\infty, \theta)=\Delta_{\infty}(\theta) / \sqrt{\omega^{2}+\Delta_{\infty}^{2}(\theta)}, \\
& \Delta(x, \theta)=\sqrt{2} \Delta(x) \cos (2(\theta-\alpha))
\end{aligned}
$$

and with the boundary condition at $\mathrm{x}=0$ derived in [10]

$$
\begin{aligned}
& k\langle g(0)\rangle \Phi_{+}^{\prime}(0)=\Phi_{+}(0)-\left\langle\Phi_{+}(0)\right\rangle \\
& \langle g(0)\rangle=\sqrt{1-\left\langle\Phi_{+}(0)\right\rangle^{2}}
\end{aligned}
$$

Here the order parameter $\Phi_{+}=(f(\theta)+f(\theta+\pi)) / 2$, where $f(\theta)$ is the Eilenberger function at the angle $\theta$ between the surface normal and quasiparticle trajectory, $v$ if the Fermi velocity, $\omega=\pi T(2 n+1)$ are the Matsubara frequencies, $x$ is the coordinate in the direction of the surface normal, $\alpha$ is the misorientation angle between the crystallographic $a$ axis and the surface normal, and angle brackets denote angle averaging. Spatial dependence of the pair-potential $\Delta$ is found from the selfconsistency equation.

\section{RESULTS AND DISCUSSION}

The boundary value problem (1) - (3) was solved numerically. The results of calculations of the angular structure of the order parameter $\Phi_{+}(x, \theta)$ are shown in Fig. 1 for two different orientations $\alpha$ of the crystallographic $a$ axis with respect to the interface normal. In both cases far from the interface the angular distribution is typical for a d-wave superconductor. However, the nucleation of a nonzero s-wave component of the order parameter $\Phi_{+}(x, \theta)$ takes place near the interface.

Indeed, as is seen from Fig.1, at $x=\xi_{0}$ the positive lobe (horizontal) is suppressed stronger than the negative one (vertical), since the characteristic length $k(\theta)$ in the direction perpendicular to the interface is small compared to $k(\theta)$ in the direction parallel to the interface. Hence at $x \approx \xi_{0}$ negative lobes of $\Phi_{+}(x, \theta)$ practically reach the local value $\Psi(x)=\Delta / \sqrt{\omega^{2}+\Delta^{2}}$ while positive ones still do not. This difference leads to the appearance of the surface-induced swave component of the order parameter $\left\langle\Phi_{+}(x, \theta)\right\rangle$, which is shown in Fig. 2. In the vicinity of the interface $\left(x \leq 0.3 \xi_{0}\right)$ the situation is just the opposite. Due to the angular dependence of $k(\theta) \propto|\cos \theta|$ the negative lobes are suppressed stronger than the positive ones, the function $\left\langle\Phi_{+}(x, \theta)\right\rangle$ changes sign to positive and reaches its maximum at $\mathrm{x}=0$.

Note that the solution $\Phi_{+}(x, \theta)$ would have pure d-wave symmetry if the pair potential $\Delta$ was spatially independent. The reason is that the characteristic length $k(\theta)$ cancels out from the solution for $\Phi_{+}(x, \theta)$ because the factor $k(\theta)$ is
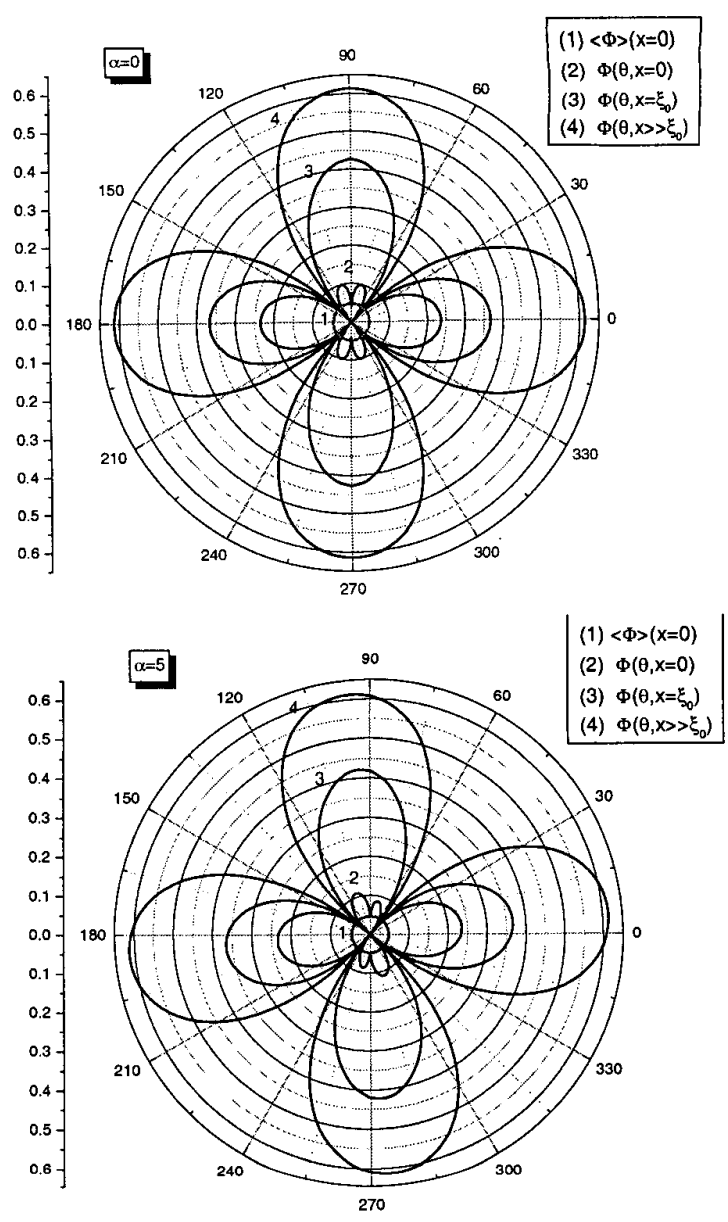

Fig.1. Angular dependence of $\Phi_{+}(x, \theta) / \pi T_{c}$ at various distances from the interface at $T=0.7 T_{c}$ for misorientation angle $\alpha=0$ and $\alpha=5^{0}$.

present both in (1) and in the boundary condition (3). However, since the pair potential is spatially-dependent, the above cancellation is incomplete and the s-wave component $\left\langle\Phi_{+}(x, \theta)\right\rangle$ nucleates at the interface.

As suggested in [4], [11] an s-wave component of the order parameter may nucleate at the surface of a d-wave superconductor if there is a subdominant bulk pairing interaction in the $\mathrm{s}$-wave channel. It is shown above that the nonzero s-wave component is localized near the rough interface even if the bulk interaction is purely $\mathrm{d}$-wave.

Fig. 3 shows the spatial variations of $\Delta(\theta)$ for different values of the angle $\alpha$. As follows from the (1) - (3) the solution for $\Phi_{+}(x, \theta)$ in the interface region has a contribution proportional to $|\cos \theta| \cos (2(\theta-\alpha))$. This in turn leads to the result that the amplitude of the s-wave component induced into the disordered layer scales with misorientation angle $\alpha$ as $\left\langle\Phi_{+}(0, \alpha=0\rangle \cos (2 \alpha)\right.$. At $\alpha=\pi / 4$ the superconducting 


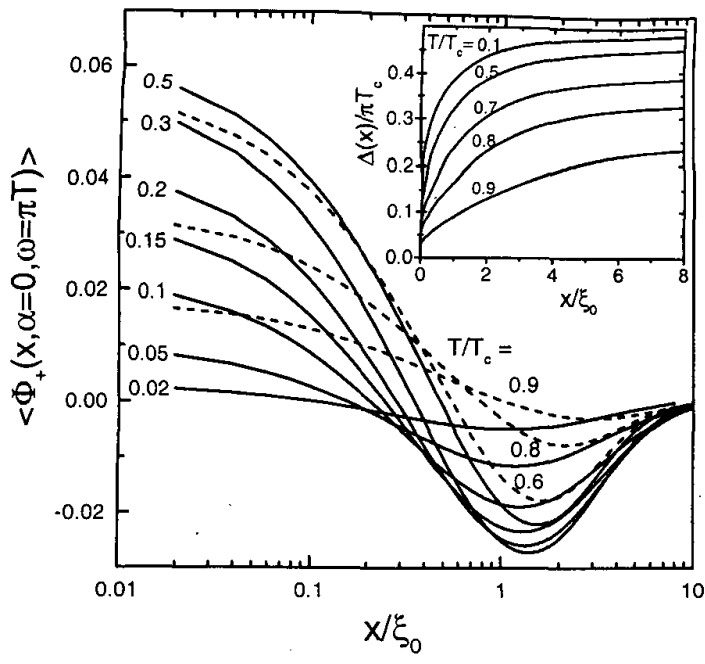

Fig.2. Spatial dependencies of the surface-induced s-wave order parameter component $\left\langle\Phi_{+}(x) / \pi T_{C}\right.$. Inset: the pair potential $\Delta(x) / \pi T_{c}$ near the interface.

correlations in the disordered layer vanish, i.e. $\left\langle\Phi_{+}(0\rangle=0\right.$. Further increase of $\alpha$ leads to a sigh change of the s-wave component.

As is seen from Fig. 3 these qualitative considerations are in a good agreement with the results of exact numerical calculations. In particular, for the $d_{x y}$ case $(\alpha=\pi / 4)$ it follows that $\left\langle\Phi_{+}(0)\right\rangle=0$. At the same time it is worth mentioning that the pair potential at the interface, $\Delta(0, \alpha=\pi / 4)$, is nonzero, in contrast to the case of a specular reflecting boundary when $\Delta$ at $\alpha=\pi / 4$ vanishes. The difference is that in the present case of diffusive scattering from the interface there is no symmetry requirement that the function $\Phi_{+}(0, \alpha=\pi / 4)$ must vanish.

In the whole temperature range the amplitude of the s-wave component $\left\langle\Phi_{+}\right\rangle$induced into the disordered layer (see Fig.2) is an order of magnitude smaller compared to the amplitude of the order parameter in the bulk superconductor. That means that $\langle g(0)\rangle$ is close to unity for all temperatures.

Thus, taking into account that $\langle g(0)\rangle$ is independent of the

Matsubara frequencies and that $\xi \Phi_{+}^{\prime}(0) \approx \Phi(0)$, we obtain from the boundary condition (3) that at low temperature and at $\omega \leq \Delta$ the function $\left\langle\Phi_{+}(0)\right\rangle \propto \omega$, while for $\omega>\Delta$ the function $\left\langle\Phi_{+}(0)\right\rangle \propto 1 / \omega^{2}$. The density of states is given by $N(\varepsilon)=N(0) \operatorname{Re}\{\langle g(0, \varepsilon=i \omega)\rangle\}$, where $\mathrm{N}(0)$ is the normal state density of states. Therefore it follows that at zero energy $N(\varepsilon=0) / N(0)=1$, i.e. the gapless superconducting state is induced in the disordered layer. This fact is due to the destructive interference along different quasiparticle trajectories

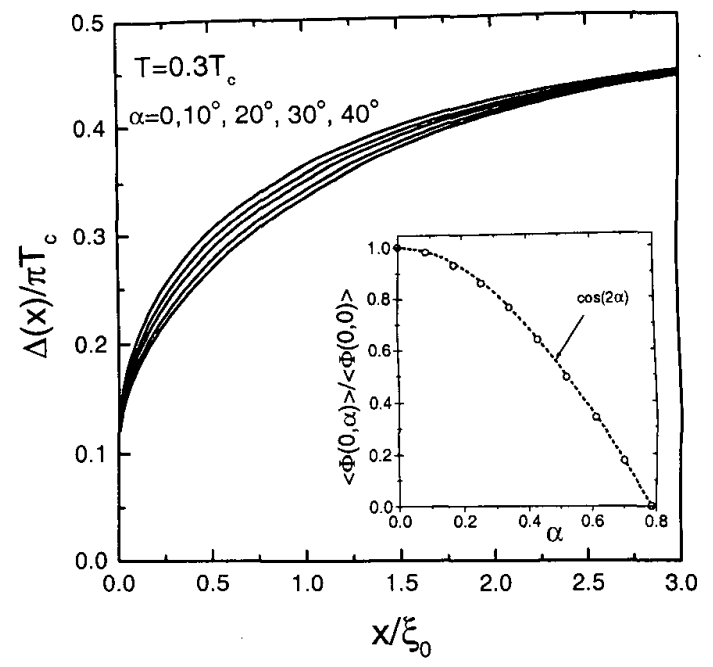

Fig.3. Behavior of the pair potential near the interface for different misorientation angles $\alpha$. Inset: dependence of $\left\langle\Phi_{+}(0)\right.$ on $\alpha$.

at $\varepsilon=0$. The averaging over the incoming trajectories yields complete vanishing of $\left\langle\Phi_{+}(0)\right\rangle$. Indeed, at $\varepsilon=0$ the magnitudes of $f(0, \theta)$ are equal for all incoming trajectories due to infinitely large coherence length $\xi=\mathrm{h} v / 2 \varepsilon$, while their phases alternate due to the $d$-wave angular structure. Thus $\left\langle\Phi_{+}(0)\right\rangle=0$ and $N(\varepsilon)=\operatorname{Re} \sqrt{1-\left\langle\Phi_{+}(0)\right\rangle^{2}}=1$.

To demonstrate this behavior explicitly we have calculated the density of states in the disordered layer by numerical integration of (1) - (3) on the real energy axis with the substitution $\omega=-i \varepsilon$ in these equations. The results of calculations of the normalized density of states for $\alpha=0$ are presented in Fig.4. It is seen that the density of states in the disordered layer is gapless and has a number of singularities at energies below the maximum bulk pair potential. These peaks are signatures of the Andreev bound states at finite energies which are due to quasiparticles trapped in the surface region with the reduced pair potential $\Delta(x)$.

Note that in the present case of extreme surface roughness there is no midgap (zero-energy) peak in the density of states in the disordered layer since incoming and outgoing trajectories are completely uncorrelated. However this peak develops for $\alpha \neq 0$ with the decrease of the degree of roughness $r$ (Fig.4). In general the quasiparticle reflection from the interface may be treated in terms of the diffusive, $r$, and the specular, $I-r$, components. The details of the calculations will be presented elsewhere. The limit $r=0$ corresponds to the case of specular interface when zero-bias peak in the density of states develops for certain values of $\alpha$ and $\theta$ (see [2], [3]).

The present model can be applied to the calculation of the Josephson supercurrent in the junctions based on d-wave superconductors (D) with rough interfaces. Let us consider 


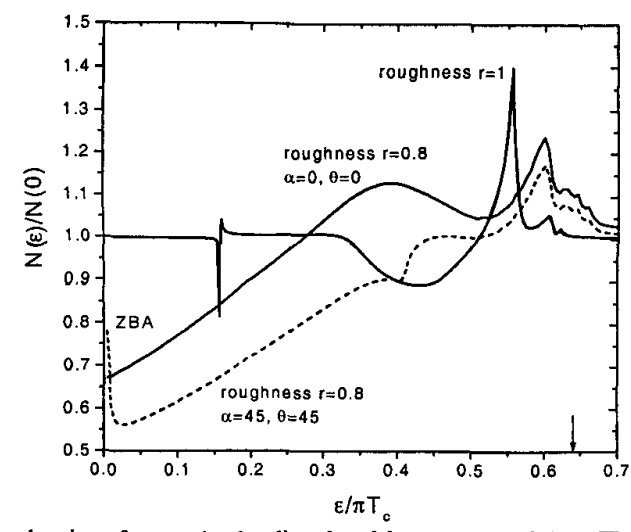

Fig.4. The density of states in the disordered layer at $T=0.5 T_{c}$. The arrow shows the maximum value of the bulk gap.

two cases: tunnel DID junction and microconstriction $\mathrm{DcD}$, where the D-layers on both sides of the weak link have rough surfaces. The critical currents in DID and DcD junctions are given by the microscopic expression in which the bulk Green's functions of the superconducting electrodes are replaced by the corresponding angle-averaged functions $\left\langle\Phi_{+}(0)\right\rangle$ and $\langle g(0)\rangle$ in the disordered layers on both sides of the barrier

$$
I_{s}(\varphi) R_{n}=\frac{4 \pi}{e t} T \underset{\omega}{\Sigma} \frac{\left\langle\Phi_{+}(0)\right\rangle^{2} \sin \varphi}{2 t^{-1}-1+\langle g(0)\rangle^{2}+\left\langle\Phi_{+}(0)\right\rangle^{2} \cos \varphi} .
$$

Here $t$ is the barrier transmission coefficient and $R_{n}$ is the normal state junction resistance. The limits $t \rightarrow 0$ and $t=1$ correspond to the DID and DcD junctions respectively.

The results of calculations are shown in Fig.5. In contrast to the conventional s-wave case both DID and DcD junctions have sinusoidal current-phase relations in the whole temperature range and equal $I_{c} R_{n}$ products because of small values of order parameters $\left\langle\Phi_{+}(0)\right\rangle$ induced in the surface layers. We note two interesting features: (a) the $I_{c} R_{n}$ products are two orders of magnitude smaller than the Ambegaokar-Baratoff result for SIS junctions, $I_{c} R_{n} / 2 \pi T_{c} \approx 0.44$; (b) the behavior of $I_{c}(T)$ is nonmonotonous. The latter property of $d$-wave junctions was predicted earlier in [12], [13] for the case of specular interfaces when it occurs for some misorientation angles $\alpha_{1,2}$. The result shown in Fig.5 corresponds to the limit of extremely strong surface roughness and does not depend on misorientation $\alpha_{1,2}$.

\section{CONCLUSIONS}

The effects of surface roughness in $d$-wave superconductors are described theoretically by introduction of a surface layer with small electronic mean free path. It is shown that the isotropic superconducting state is realized in such a layer. The surface density of states is gapless and exhibits a number

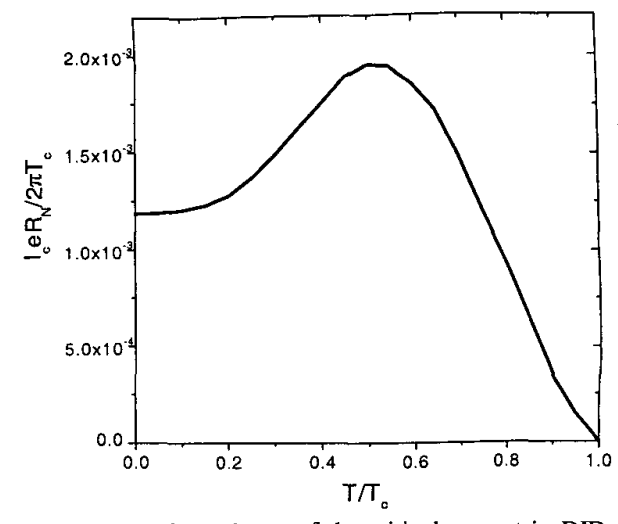

Fig.5. The temperature dependence of the critical current in DID and DcD Josephson junctions with rough interfaces.

of finite bias peaks, while the midgap peak appears only at smaller roughness parameter. The nonmonotonous temperature dependence of the critical current in DID and DcD Josephson junctions with rough interfaces is predicted.

\section{ACKNOWLEDGMENT}

The authors would like to thank H.Rogalla, G.Gerritsma and Yu.Nazarov for helpful discussions.

\section{REFERENCES}

1. C.- R. Hu, "Midgap surface states as a novel signature for d-wave superconductivity", Phys. Rev. Lett., vol. 72, pp. 1526-1529, 1994.

2. Y. Tanaka and S. Kashiwaya, "Theory of tunneling spectroscopy of dwave superconductors", Phys. Rev. Lett., vol. 74, pp. 3451-3454, 1995.

3. Yu. S. Barash, A. A. Svidzinsky and H. Burkhardt, "Quasiparticle bound states and low-temperature peaks of the conductance of NIS junctions in dwave superconductors", Phys. Rev. B, vol.55, pp. 15282- 15293, 1997.

4. M. Fogelstrom, D. Rainer and J. A. Sauls, "Tunneling into currentcarrying surface states of high $T_{c}$ superconductors", Phys. Rev. Lett., vol. 79, pp. 281-284, 1997.

5. L. Alff, H. Takashima, S. Kashiwaya, et al., "Spatially continuous bias conductance peak on (110) YBCO surfaces", Phys. Rev. B, Vol.55, pp.R14757 - R14760, 1997.

6. M. Covington, M. Aprili, L. H. Greene, F. Xu, J. Zhu and C. A. Mirkin, "Observation of surface-induced broken time-reversal symmetry in YBCO tunnel junctions”, Phys. Rev. Lett., vol. 79, pp. 277-280, 1997.

7. F. J. Culetto, G. Kieselmann and D. Rainer, in Proc. of the 17th International Conf. on Low Temperature Physics, U. Eckern, A. Schmid, W. Weber and H. Wuhl, Eds. Amsterdam: North Holland, 1984, pp. 1027-1028.

8. Yu. N. Ovchinnikov, "Critical currrent of superconducting films with diffusive surface scattering", Sov. Phys. JETP, vol. 29, pp. 853-861, 1969.

9. G. Eilenberger, "Transformation of Gorkov's equations for type II superconductors into transport-like equations", Z. Physik, vol. 214, pp. 195-208, 1968.

10. A. A. Golubov and M. Yu. Kupriyanov, "Anomalous proximity effect in d-wave superconductors", JETP Lett., vol.67, pp. 501-507, 1998.

11. S. R. Bahcall, "Boundary effects and the order parameter symmetry of high $T_{c}$ superconductors", Phys. Rev. Lett., vol. 76, pp. 3634-3637, 1996.

12. Y. Tanaka and S. Kashiwaya, "Theory of Josephson effects in anisotropic superconductors", Phys. Rev. B., vol. 56, pp.892-912, 1997.

13. Yu. S. Barash, H. Burkhardt and D. Rainer, "Low-temperature anomaly in the Josephson critical current of junctions in d-wave superconductors", Phys. Rev. Lett., vol. 77, pp. 4070-4073, 1996. 